\title{
La géographie humaine d'Hydace
}

The Human Geography of Hydatius

\section{Christian Stein}

\section{(2) OpenEdition}

Journals

Édition électronique

URL : https://journals.openedition.org/gaia/549

DOI : $10.4000 /$ gaia. 549

ISSN : 2275-4776

\section{Éditeur}

UGA Éditions/Université Grenoble Alpes

\section{Édition imprimée}

ISBN : 978-2-37747-199-7

ISSN : $1287-3349$

\section{Référence électronique}

Christian Stein, «La géographie humaine d'Hydace », Gaia [En ligne], 22-23 | 2020, mis en ligne le 01 juin 2020, consulté le 09 décembre 2021. URL : http://journals.openedition.org/gaia/549 ; DOI : https://doi.org/10.4000/gaia.549

Ce document a été généré automatiquement le 9 décembre 2021.

Gaia. Revue interdisciplinaire sur la Grèce archaïque 


\title{
La géographie humaine d'Hydace
}

\author{
The Human Geography of Hydatius
}

\section{Christian Stein}

1 Quelle était la géographie humaine des Romains de l'Antiquité tardive ? Si, de nos jours, parler de géographie fait d'abord surgir à l'esprit une multitude d'images de cartes de toutes natures, la situation était bien différente dans l'Antiquité. Cartes et itinéraires existaient, certes, mais restaient rares et approximatifs. Pour ce qu'on en sait, le mode principal de transmission et de représentation des connaissances géographiques dans l'Antiquité n'était pas une quelconque forme iconographique mais le traité qui allait décrire par des mots les espaces et les populations considérés, les Romains ayant aussi un goût prononcé pour la représentation linéaire de l'espace à travers des itinéraires dont la table de Peutinger est un écho, puisqu'elle montre plus des routes que des espaces ${ }^{1}$.

2 En quelques pages, l'ambition de cet article ne peut évidemment être que réduite : on se bornera donc ici à un sondage très partiel, centré sur un auteur occidental tardif et donc latin, Hydace, agrémenté de quelques comparaisons avec certains de ses contemporains. La finalité sera purement descriptive : elle consistera à tenter de saisir dans quel cadre Hydace percevait l'espace romain de son temps ainsi que les populations qui y résidaient et, si possible, de voir en particulier quelle place y tenaient la cité et/ou le fait urbain.

\section{Hydace}

Avant d'aller plus loin, il convient toutefois d'abord de présenter succinctement notre source principale, qui est en effet assez peu connue hors du cercle restreint des tardoantiquisants, en particulier en France ${ }^{2}$. Cela permettra ainsi de mettre en relief l'intérêt qu'il présente pour l'étude de la cité et du fait urbain en général dans l'Antiquité.

Hydace ne nous est connu que par les informations qu'il a lui-même glissées dans son œuvre et qui sont toujours incidentes. Il est originaire et a passé l'essentiel de son existence au nord-ouest de la péninsule Ibérique. Il est né très probablement vers 
395-400 dans la cité de Lemica, au milieu de la province de Galice, à cheval entre l'Espagne et le Portugal actuels, pour y mourir dans les années 470, pense-t-on ${ }^{3}$. Il a voyagé en Orient dans sa jeunesse, puisqu'il écrit avoir vu Jérôme en Terre sainte, peutêtre vers 406-407, avant d'accéder en 427 à l'épiscopat à Aquae Flaviae, l'actuelle Chaves, toujours en Galice, à une quarantaine de kilomètres à peine au sud de sa cité d'origine. On en déduira donc, sans grande crainte de se tromper, qu'il était issu d'une famille indiscutablement aisée et bien ancrée dans sa région, mais sans plus de précisions sur son contexte. Ce qui est assuré, en revanche, est qu'il fut un témoin direct de la désagrégation de l'Empire romain en Occident, donc un témoin de l'effacement d'une bonne partie des structures impériales dans des conditions dramatiques au ve siècle. Il y a un débat moderne dans le milieu des spécialistes de l'Antiquité tardive entre les historiens qui pensent que la fin de l'Antiquité fut brutale, assimilable à un effondrement au sens popularisé par l'anthropologue et géographe Jared Diamond, et ceux qui estiment qu'elle fut, sinon heureuse, du moins tellement segmentée qu'elle en aurait été presque indolore ${ }^{4}$. Il n'y a pas de doute à avoir sur le fait qu'Hydace aurait rejoint les premiers, lui qui vécut aux premières loges l'irruption des Suèves et Vandales puis des Goths en péninsule Ibérique à partir de 409, et plus particulièrement en Galice à partir de 411. Le choc dut être particulièrement rude car, autant les provinces frontalières du Rhin ou du Danube étaient relativement coutumières des incursions dites «barbares » depuis la fin du II $^{\mathrm{e}}$ siècle, autant les populations du sud des Pyrénées vivaient dans une sorte de havre qui n'avait été troublé jusque-là que de manière très sporadique ${ }^{5}$.

5 Si Hydace bénéficie encore aujourd'hui d'une certaine notoriété, c'est parce qu'on a conservé de lui une unique et précieuse Chronique ${ }^{6}$. Il la composa dans la continuité de celle d'Eusèbe, qui s'arrête en 325, elle-même prolongée par Jérôme qui va jusqu'en 378. Débutant en 379, la chronique d'Hydace s'interrompt en 468, sans doute peu de temps avant sa mort? ${ }^{7}$.

6 Hydace constatait certainement aussi que les échanges avec le reste de l'espace romain allaient en s'amenuisant à mesure que l'Empire se désagrégeait. Tout cela se ressent dans son œuvre, qui n'est pas du tout focalisée sur un centre impérial habituel, comme l'Italie/Rome ou Constantinople, mais bien sur cette Galice où il résidait. Quand on fait le compte de la fréquence des lieux et espaces cités par Hydace, sa province remporte la palme, sans compter la profusion de tous les lieux situés dans la province elle-même et qu'il mentionne aussi. Viennent ensuite l'ensemble des provinces et villes ibériques, puis les références au sud-ouest de la Gaule. Pour le reste de l'Empire, les mentions sont beaucoup plus rares. Cela réduit évidemment la portée générale de la Chronique, qui n'informe finalement bien que sur les événements se déroulant dans la péninsule Ibérique au ve siècle. En ce sens c'est aussi intéressant pour nous, parce qu'il n'est pas le porte-parole d'un centre impérial mais bien plutôt celui d'une périphérie de plus en plus marginale. Il écrivit en effet cette chronique depuis une province qu'il concevait lui-même comme une sorte de bout du monde occidental de l'Empire, cet état d'esprit étant probablement alimenté par le fait qu'il avait vu et traversé ce dernier pour aller en Orient ${ }^{8}$.

7 L'objectif que nous nous assignerons sera alors de relever l'ensemble des allusions géographiques et topographiques dans sa Chronique pour déterminer quel cadre conceptuel Hydace propose à son lecteur pour appréhender l'espace impérial, avec en particulier le rôle qu'y joue le fait urbain. L'ambition ne sera pas de discuter le degré 
de véracité des propos d'Hydace en ce qui concerne l'histoire événementielle, mais plutôt quelle carte imaginaire de l'Empire il laisse à son lecteur' .

\section{Mise en contexte : la ciuitas romaine}

8 À ce stade, il convient toutefois de donner quelques éléments de contexte afin de remettre la géographie humaine d'Hydace en perspective. Dans le monde romain d'époque classique, l'unité de référence de la géographie humaine était avant tout la cité. L'espace romain en formation était d'abord un monde de cités qui entrèrent progressivement dans l'orbite romaine, que ce soit sous forme amicale ou inamicale via la conquête : cela donne grossièrement d'un côté les cités dites libres ou fédérées, de l'autre les tributaires (ou stipendiaires). Entre ou autour de ces cités évoluaient cependant aussi parfois des peuples qui n'avaient pas droit à l'appellation de cité, mais qui le devenaient progressivement sur le long terme.

9 Évidemment, cela pose la question de savoir exactement ce qu'était une cité et ce qu'il faut aujourd'hui mettre derrière le terme de ciuitas. La traduction de ciuitas par " cité », si elle est commode et omniprésente, n'explique en effet pas grand-chose quand on y repense, car il s'agit d'une simple transposition. L'enfer réside alors non dans la traduction proprement dite des termes anciens, mais bien dans leur interprétation ${ }^{10}$.

10 Ce n'est pas ici qu'on se lancera dans un long débat sur la nature de la ciuitas, débat à mettre évidemment en parallèle avec tout ce qui s'est fait sur la nature de la polis grecque autour de Mogens Hansen et du Copenhagen Polis Center ${ }^{11}$. On pourra cependant s'arrêter sur plusieurs grands critères généraux qui caractérisent la conception romaine de la cité et qui devraient être assez consensuels. Le premier est qu'une cité est d'abord une communauté humaine, communauté animée par une vie civique fondée sur l'idée de participation libre des citoyens, cette communauté étant autonome et fixant elle-même ses règles. Tout ceci se déroule ensuite sur un territoire qui est organisé par l'occupation humaine, avec au cœur de ce territoire un pôle urbain, une ville qui est presque toujours métonymique de la cité.

11 D'un point de vue de géographe actuel, on pourrait d'une certaine manière considérer que ciuitas sert à désigner une sorte de "fait urbain total ", avec l'intégration du bâti, de son territoire, de son hinterland, des populations qui résident là et sont actrices d'une vie communautaire et culturelle spécifique, mais aussi l'intégration des relations de ce «fait urbain total» avec les entités humaines voisines. On préfèrera ici cette forme d'interprétation de ciuitas à l'assimilation assez fréquente de cette dernière avec notre concept d'État qui est sans doute trop moderne.

12 Mais, tout ce qui précède correspond évidemment à la théorie, car, dès la fin de l'époque républicaine, la constitution de l'empire, qui devint ensuite un Empire, altéra significativement les caractéristiques pratiques de cette définition. Pour aller vite, on dira que dans un premier temps qui dura jusqu'au $\mathrm{I}^{\mathrm{er}}$ siècle av. J.-C., la cité romaine phagocyta progressivement toutes les autres cités et peuples italiens. Le territoire romain devint ainsi gigantesque (d'un point de vue antique), avec une ville de Rome qui devint un pôle quasi macrocéphalique, tandis que toutes les anciennes villes-capitales rétrogradaient au rang de pôles urbains secondaires, après une histoire locale parfois compliquée, comme ce fut le cas de Capoue. Dans un deuxième temps, cette évolution italienne se prolongea avec l'intégration de l'ensemble des cités de l'empire à la (de 
plus en plus) monstrueuse " cité » romaine, dans un processus qui s'étala sur plus de deux siècles. Un processus spatial qui passa presque toujours d'abord par l'installation de colonies romaines provinciales, puis l'intégration de pérégrins isolés, puis l'intégration de communautés pérégrines entières. À nouveau, les villes qui étaient au cœur de ces cités perdirent leur rang de villes-capitales, mais en même temps, comme on était beaucoup plus loin de la géante et tentaculaire ville de Rome, beaucoup de ces villes désormais romaines et secondaires - mais en même temps, comparées à Rome n'importe quelle ville de l'époque était secondaire - virent en réalité leur taille et leur rayonnement augmenter dans le cadre de la pax Romana.

Dès le $\mathrm{I}^{\mathrm{er}}$ siècle av. J.-C. le modèle théorique de la cité était donc dépassé, et c'est Cicéron qui a théorisé cela pour l'Italie avec le modèle des deux patries: tous les Romains appartiennent à la grande patrie romaine qui est leur patrie de droit, et en même temps ils sont membres d'une patrie de rang inférieur, leur petite patrie, où ils sont nés ou celle de leurs racines, qui devient en quelque sorte une cellule du grand ensemble romain ${ }^{12}$. Et ce modèle se diffusa ensuite à l'époque impériale hors d'Italie : on retrouve encore cette façon de concevoir les choses à l'époque tardive chez Ausone, qui revendique Rome et Bordeaux comme ses deux patries, ou chez Rutilius Namatianus, qui clame son appartenance à la communauté romaine mais quitte la ville de Rome pour revenir aider sa patrie gauloise dont le nom est malheureusement inconnu ${ }^{13}$.

14 À ce stade de l'enquête, on remarquera que la traduction quasi systématique du latin ciuitas par l'immuable « cité » est finalement un faux-ami car les réalités sous-jacentes sont très différentes selon que l'on se place, par exemple, en 200 avant ou en 300 après J.-C. On pourrait certes garder le terme de « cité » pour interpréter la ciuitas de l'époque de Scipion l'Africain, mais il serait alors préférable de trouver un autre mot français pour désigner la ciuitas du temps de Dioclétien. À tout prendre, s'il fallait faire une proposition, il me semble que dans notre français actuel le mot "commune » serait alors plus adapté $e^{14}$.

De fait, cette proposition de traduire différemment en français actuel le mot ciuitas selon son époque d'emploi ne devrait avoir rien de révolutionnaire. Dans l'exemple proposé, entre Scipion et Dioclétien, l'écart chronologique est d'un demi-millénaire. Cela ne surprendrait personne si on disait qu'en français actuel une grande partie des mots existant déjà il y a cinq siècles et toujours en usage aujourd'hui n'ont plus exactement le même sens. Alors pourquoi voudrait-on qu'il en fût autrement en latin ? Certes les langues n'évoluent pas toutes de la même manière linéaire, mais sur une période aussi longue et avec des contextes socio-politiques aussi différents, il n'y a guère de doute à avoir sur la pertinence de la démarche.

De manière latérale, on pourra d'ailleurs aussi observer que pour l'interprétation de la polis grecque, souvent assimilée un peu vite à la ciuitas romaine, les choses ont évolué de manière assez différente, dans la mesure où le monde grec ne vit jamais se développer un objet aussi hors norme tel que Rome, même s'il connut quand même aussi les royaumes hellénistiques. Sur la longue durée, le cadre conceptuel grec de la polis resta donc plus homogène que le cadre conceptuel latin de la ciuitas, sauf évidemment pour les Grecs qui tentèrent de considérer Rome à la mode grecque ${ }^{15}$. 


\section{Complexification et hiérarchisation des cadres humains}

Dans ce cadre, on peut donc concevoir le monde romain comme un agrégat croissant de cités. Néanmoins, si la cité était l'unité humaine de base de la géographie humaine romaine, pour gouverner et administrer tout cela, le pouvoir central romain ne pouvait pas se permettre le luxe d'un dialogue direct et constant avec la myriade de cités composant l'empire en expansion : cela eût été concrètement impossible. Dès l'époque républicaine, mais plus encore à l'époque impériale, la géographie humaine romaine s'est donc enrichie de plusieurs niveaux, plusieurs strates d'échelles différentes, destinées à mieux articuler le centre et ses périphéries, lesquels ont évidemment à nouveau altéré la cité et réduit son influence et son rôle.

On pourrait considérer un peu rapidement que ces niveaux qui se sont intercalés entre les cités et le pouvoir central ne relèveraient que de la géographie administrative, puisqu'il s'agit de ces entités que nous connaissons sous le nom de "provinces", " diocèses ", " préfectures du prétoire ", etc., mais ce point de vue administratif serait trop réducteur. Les autels provinciaux ou régionaux du culte impérial ainsi que leur fonctionnement, comme l'autel des Gaules situé juste à côté de la colonie de Lugdunum, montrent l'émergence de formes de communautés provinciales qui n'étaient sans doute pas que des structures purement artificielles. Au ve siècle, un Rutilius Namatianus s'identifiait très fortement comme Gaulois, ce qui n'aurait pas vraiment eu de sens à l'époque de César ${ }^{16}$.

Or c'est exactement ces processus qu'on retrouve clairement chez Hydace. Quand on lit sa Chronique, on constate bien en effet une logique générale de l'organisation de l'espace romain avec, pour l'essentiel, des structures qui s'emboîtent les unes dans les autres. Du plus vaste au plus petit, on retrouve alors plusieurs niveaux correspondant chacun à une échelle spatiale :

1. Au niveau le plus élevé et le plus vaste on a évidemment l'Empire en entier, l'imperium Romanum, qui apparaît à plusieurs reprises exprimé sous une forme ou une autre (p. ex. § 7).

2. Au niveau d'en-dessous, cet Empire se divise entre ses parties occidentale et orientale (p. ex. $\S 143=$ M151, qui distingue en 451 des prodiges visibles dans la part orientale de ceux visibles dans la part occidentale).

3. Viennent ensuite les grandes régions que sont l'Espagne (Hispanias), la Gaule, l'Italie, l'Afrique, l'Égypte. Les autres sont absentes, mais on se rappellera que notre point de vue est situé en Galice. Il n'est toutefois pas certain que ces régions soient réductibles chez Hydace aux circonscriptions administratives qu'étaient les diocèses ou les préfectures du prétoire portant les mêmes noms.

4. Viennent ensuite quelques provinces peu nombreuses. Hydace mentionne les cinq de la péninsule Ibérique à son époque, mais ne va guère au-delà. Il évoque bien l'Aquitaine (Aquitanica), depuis Toulouse jusqu'à la mer $(\S 61=\mathrm{M} 69)$, regroupant sans doute à cette occasion les différentes provinces portant ce nom. Il mentionne aussi la Maurétanie envahie par Genséric $(\$ 80=\mathrm{M} 90)$, mais sans plus de précision.

Le niveau provincial n'est toutefois pas toujours pertinent pour lui et il lui préfère le niveau régional. Quand il veut aider le lecteur à situer une cité éloignée, il ne la place pas dans sa province, mais dans son ensemble régional. Ainsi, Martin est évêque de Tours in Gallis, « dans les Gaules» ( $\$ 8)$, ou encore la cité de Béziers, in Gallicana regione ( $\$ 65=$ M73). Une autre hypothèse, parfaitement envisageable, serait aussi qu'Hydace ne connaissait pas bien les limites des provinces hors celles de péninsule Ibérique. 
5. Au niveau inférieur, Hydace évoque également à plusieurs reprises les trois conventus de Lugo, Braga, Astorga, districts judiciaires qui sont tous situés dans la province de Galice (p. ex. $\S 93=$ M102, § $172=$ M179).

6. Ensuite vient le niveau des ciuitates et/ou des villes, niveau avec le plus de mentions et sur lequel on reviendra plus loin : ce sont ces structures qui sont le plus souvent évoquées dans la Chronique.

7. Enfin, au niveau inférieur à celui de la ville/cité, peut-être assimilable à une sorte de niveau infra-urbain, on retrouve plusieurs types de formes humaines d'organisation. Il y a de rares mentions de castella ( $(41$ et $81=\mathrm{M} 49$ et 91$)$ et de castra ( $\$ 179$ et $190=186$ et 195) qui correspondent certainement à des lieux fortifiés ou en tout cas à identité militaire marquée. Il y a aussi des loca, terme qui désigne le plus souvent des endroits précis dans le cours de la narration, mais aussi parfois des localités certainement non militarisées, et éventuellement qualifiées camporum ou maritima (p. ex. §164= M171, §168= M175, §197= M202). Il y a même une mention de uillae en Galice $(\S 213=\mathrm{M} 213 \mathrm{a})$ : s'agit-il encore là de grandes exploitations rurales ou déjà de proto-villages ?

Notons qu'à l'échelle de l'Empire, certains lieux particuliers apparaissent également en tant que tels : le Palais impérial qui peut être localisé $(\S 95=$ M104) ou pas $(\S 70=$ M78), les Lieux saints de Jérusalem (Praef., 4, § 58 = M66), le Champs de Mars à Rome (§ $154=$ M162).

Sans surprise, on a dans ce qui précède, l'ensemble de la géographie administrative de l'Empire tardif, y compris certains lieux emblématiques du pouvoir ou de la religion, et on retrouve en partie cette stratification dans l'identité des personnes. Quand Hydace introduit dans son récit l'empereur Théodose, il l'identifie comme Theodosius, natione Spanus de provincia Gallaecia ciuitate Cauca, "Théodose, Espagnol de nation, de la province de Galice, de la cité de Cauca » $(\S 2)$ : on retrouve là trois de ces niveaux successifs, la région, la province et la cité. Quand dans sa préface il se présente luimême, il se décrit de la province de Galice né dans la cité des Lemici, Ydatius provinciae Galleciae, natus in Lemica ciuitate (Praef., 1). Mais il s'agit dans ces deux exemples de Galiciens et dès qu'on s'éloigne de la province Galice, la ciuitas s'efface: le prêtre Germanus est presbiter Arabicae regionis $(\S 97=$ M106), de la "région arabique »; l'apostat Ajax, natione Galata, «de nation galate» $(\$ 228=$ M232). L'identité civique a donc du sens pour définir des individus proches, mais elle perd sa pertinence pour évoquer une origine plus lointaine.

21 Toute cette structure, en particulier en Gaule et en péninsule Ibérique, est cependant perturbée par l'irruption des peuples barbares qu'on voit se mouvoir au sein de l'Empire, et en particulier en péninsule Ibérique. Ces barbares s'approprient des portions de territoire au sein des différentes strates qui viennent d'être évoquées. Ils parasitent l'organisation des pouvoirs en instituant un concurrent au pouvoir romain. Le regnum caractérise ainsi chez Hydace d'abord l'autorité et le pouvoir des Augustes romains ( $\$ 1$, 9 , etc.), mais aussi le pouvoir des rois barbares comme Alaric, Athaulf ( $\$ 37=$ M45), Vallia ou Theoderic $(\S 62=$ M70), etc. Un pouvoir qui est cependant fragile, comme le démontre la destruction du regnum suève $(\$ 168=$ M175).

\section{Nommer le fait urbain tardif}

Ce tableau général ayant été dressé, il est maintenant possible de revenir vers le niveau qui nous intéresse le plus ici, celui qui désigne le fait urbain à travers la ville ou la cité. Dans sa Chronique, Hydace utilise en effet parfois le terme urbs, plus souvent il écrit ciuitas, et plus souvent encore il utilise directement un nom propre qui se suffit à lui- 
même, en particulier quand il nomme un évêque, comme Sabinus, évêque d'Hispalis, Sabinus, episcopus Ispalensis ( $\$ 187=$ M192a).

De manière générale, on a toutefois largement l'impression que ces termes sont chez Hydace interchangeables et ont le même sens, à moins qu'ils ne recouvrent des nuances qui nous sont difficilement perceptibles ${ }^{17}$. Dans deux passages à peine éloignés de quelques lignes et dans lesquels il est clairement fait allusion à des événements concernant des agglomérations, Hydace parle de la «ville » (urbs) d'Astorga ( $\$ 166=$ M173) puis la « cité » (ciuitas) de Braga $(\$ 167=$ M174), sans qu'on arrive à déterminer pourquoi il a choisi un mot plutôt que l'autre.

24 Néanmoins, quand on regarde dans le détail, il apparaît tout de même que cette indifférenciation n'est pas entièrement parfaite. Les occurrences du terme urbs montrent ainsi que son utilisation est assez réduite et plutôt particulière puisque si le terme apparaît treize fois dans la Chronique, six désignent Rome, visiblement l'urbs par excellence ${ }^{18}$.

Le terme ciuitas est en revanche utilisé de manière plus indifférenciée, avec dix-huit occurrences dont, fait révélateur, aucune à propos de la Ville éternelle ${ }^{19}$. Ce qui est alors très frappant quand on compare ces occurrences, c'est qu'Hydace insère presque toujours la ciuitas dans une entité supérieure qui peut être la province mais aussi une région. On l'a déjà vu faire quand il décline son identité ou celle de Théodose, mais un peu plus loin il écrit : In provincia Cartaginiensi in ciuitate Toleto... ( $25=\mathrm{M} 31$ ), In Gallicana regione in ciuitate Biterris... ( $(65=\mathrm{M} 73$ ), etc. Il est très rare qu'Hydace mentionne une ciuitas comme entité autonome, elle est toujours une subdivision de quelque chose de plus grand et d'englobant.

Constatant cela, avec notre regard spatial moderne, on serait alors en droit de penser qu'on retrouve là une vision de l'espace où la ciuitas ressemble à la cité classique, un espace régional donné étant subdivisé en ciuitates au centre desquelles on trouverait à chaque fois une ville/urbs principale. Néanmoins, plusieurs des occurrences montrent qu'Hydace ne conçoit pas l'espace de la sorte. Si la plupart sont de sens ambigü, ces occurrences-là montrent en effet indubitablement que lorsqu'il utilise le terme ciuitas, il pense plutôt à la ville au sens matériel d'une agglomération, sans doute cernée d'une enceinte, osera-t-on deviner. Cela apparaît évident quand il écrit qu'en 457 les Suèves prennent par ruse la ville de Lisbonne : ciuitas Ulixippona sub specie pacis intratur ( $\$ 181=$ M188). Cela est encore plus flagrant lorsqu'il décrit, dans un long paragraphe, la prise d'Astorga la même année par Théodoric. Le schéma est classique, mais la description s'achève par un parallèle entre les maisons de la ciuitas qui sont incendiées et les loca camporum qui sont dévastés ${ }^{20}$ : on retrouve bien là un tandem essentiellement topographique ville/localités rurales (puisqu'il est un peu trop tôt pour déjà parler de villages).

27 Tout ceci amène donc à l'idée qu'Hydace conçoit la ciuitas non comme un espace mais plutôt comme un point remarquable dans une aire provinciale ou régionale dont il n'évoque finalement pas grand-chose d'autre en termes d'habitat humain: les mentions de localités et autres castra sont résiduelles. Dans le même temps, appeler cette ville ciuitas semble être plus valorisant que la qualifier d'urbs, terme qui reste en retrait sauf pour désigner la lointaine Rome ${ }^{21}$. Hydace est donc finalement encore assez ancré dans une vision romaine traditionnelle des choses, qui n'accorde véritablement d'importance et de visibilité qu'aux villes et non aux campagnes ${ }^{22}$. 
$28 \mathrm{Au}$ niveau des agglomérations, on peut également faire une autre remarque topographique: Hydace fait très rarement allusion à des éléments d'architecture urbaine. À titre de comparaison, on notera que ces caractéristiques ne correspondent pas du tout à ce qu'on retrouve chez un contemporain direct d'Hydace, Paulin de Pella (376/377-après 460) qui publia son autobiographie vers 459 et qui était un quasi voisin, puisqu'il vivait alors entre Bordeaux et Marseille. Paulin n'utilise pas le terme de ciuitas, mais toujours urbs ou un nom propre, et quand il doit parler ou décrire une ville, il fait très vite allusion à son enceinte. Il naît à Pella prope moenia Thessalonices, " près des remparts de Thessalonique", de là il va moenia Sidoniae Carthaginis usque, "jusqu'aux murs de Carthage la Sidonienne », avant de continuer jusqu'aux remparts de Rome, etc. ${ }^{23}$. Une bonne question serait de savoir si entre Paulin et Hydace la différence tient à deux individus qui ont chacun leur manière de présenter la ville, ou à deux modes d'expression différents: Hydace fait une chronique, tandis que l'autobiographie de Paulin est rédigée selon les canons de la poésie. Cela signifie probablement que le fait urbain ou la ville ne se désigne pas de la même manière selon la nature de la source écrite. Mais est-ce alors seulement une affaire de métonymie?

La caractéristique la plus saillante de la géographie qu'Hydace lègue à la postérité tient cependant à la christianisation de l'espace qu'il propose, en particulier concernant les villes.

On le distingue à deux éléments. Le premier est d'observer le nombre de villes (ou cités) qui sont essentiellement associées à leur évêque : il est considérable, et beaucoup de villes (ou cités) n'apparaissent dans la géographie hydacienne que grâce à leur évêque. D'une certaine manière, si aucun évêque n'y réside, elles n'existent pour ainsi dire pas : la présence d'un évêque assure la notoriété d'une agglomération comme le faisait la résidence d'un gouverneur ou d'une préfecture du prétoire. Le cas extrême est celui de Rome puisque plus de la moitié des évocations de la Ville par excellence de l'Empire correspondent uniquement à l'indication de l'avènement d'un nouvel évêque. C'est d'ailleurs le seul élément qui montre encore l'importance de Rome, car c'est l'unique ville qui bénéficie de cette attention, malgré son éloignement de la Galice. Ce qui fait l'importance d'une ville chez Hydace n'a donc rien à voir avec sa dimension proprement urbaine, mais presqu'entièrement avec l'aura de son siège épiscopal ou l'aura personnelle remarquable de ses évêques.

31 L'autre élément de christianisation de l'espace se retrouve au niveau de la topographie urbaine. Nous avons dit qu'Hydace ne s'intéressait pas aux éléments architecturaux des villes, mais il faut maintenant corriger un peu le propos. Il mentionne pour quelques villes peu nombreuses des lieux saints particulièrement efficaces, mais qui ne se confondent pas avec toutes les églises en général : on n'en est pas encore à l'époque où le lieu de culte qu'est l'église prend la place sacrée qu'il aura à partir des $\mathrm{VIII}^{\mathrm{e}}-\mathrm{XII}{ }^{\mathrm{e}}$ siècle ${ }^{24}$. Il s'agit plutôt de sanctuaires chrétiens spécifiques qui abritent les restes de quelques saints personnages, comme les tombeaux de saint Pierre et saint Paul à Rome et que la troupe d'Alaric épargne lors du sac de Rome ( $\$ 35=$ M43). Mais il peut aussi s'agir de la profanation d'un sanctuaire comme l'église de Mérida consacrée à sainte Eulalie, ce qui vaut au profanateur sa perte $(\S 80=\mathrm{M} 90)$. Dans l'imaginaire hydacien on retrouve donc tous les arguments qui vont amplifier cette tendance chrétienne à inclure dans les agglomérations humaines les reliques et autres objets liés aux saints afin de bénéficier de leur aura protectrice (ou vengeresse). Dans ce monde qui se christianise progressivement, l'espace des morts, ou tout au moins de certains morts, 
commence à se rapprocher de celui des vivants, en particulier dans la ville. Cette villelà, de plus en plus chrétienne, s'éloigne alors du cadre antique classique qui justement maintenait fermement les morts à l'écart de la ville des vivants. Bref, on n'en est pas encore au «blanc manteau d'églises » qui caractérisait l'Europe imaginaire de Raoul Glaber au $\mathrm{XI}^{\mathrm{e}}$ siècle (Histoires, II, 4), mais on voit bien que le mouvement est sur sa lancée.

\section{Incertitudes}

Pour clore cette courte description du monde d'Hydace et prolonger nos interrogations sur le vocabulaire et les concepts antiques ainsi que leur réception par nous aujourd'hui, il est difficile de ne pas constater le fait que ces questions sont en réalité très anciennes, ce qui nous incitera à penser que les agaçantes zones de flou ne sont pas prêtes à se dissiper.

Quand il écrivit au milieu du $\mathrm{VI}^{\mathrm{e}}$ siècle son histoire universelle improprement appelée aujourd'hui Histoire des Francs, Grégoire de Tours raconta en effet dans un passage l'histoire d'un saint homme résidant dans la ville (urbs) de Langres et évêque de Dijon. Grégoire se saisit de l'occasion pour insérer une petite digression sur Dijon dont il décrivit le site ainsi que son castrum, et il se sentit alors obligé de faire partager à son lecteur une question qui le dérangeait visiblement beaucoup. Alors que Dijon en avait à ses yeux toutes les caractéristiques, il ne comprenait pas pourquoi il n'était pas d'usage d'utiliser le terme de ciuitas pour qualifier ce lieu : Qui cur non ciuitas dicta sit, ignoro ${ }^{25}$.

\section{BIBLIOGRAPHIE}

\section{Éditions de la Chronique}

BURGESS Richard W., The Chronicle of Hydatius and the Consularia Constantinopolitana. Two Contemporary Accounts of the Final Years of the Roman Empire, édition traduite en anglais, Oxford, Clarendon Press, 1993.

MOMMSENN Theodor (éd.), Hydatii Lemici continuatio chronicorum Hieronymianorum, MGH, AA, Chronica minora, II, t. XI, Berlin, Apud Weidmannos, 1894, p. 3-36.

SCÖTTER Jan-Marcus \& SCARDINO Carlo, Chronik des Hydatius. Fortführung der Spanischen Epitome, édition traduite en allemande et commentée, Paderborn, Ferdinand Schöningh, 2019.

TRANOY Alain, Hydace, Chronique, I : Introduction, texte critique, traduction, II : Commentaire et index, Paris, Le Cerf, 1974 (coll. « Sources chrétiennes », $\mathrm{n}^{\text {os }} 218$ et 219).

\section{Études}

ALBU Emily, « The Battle of the Maps in a Christian Empire », dans C. Rapp \& H. A. Drake (éd.), The City in the Classical and Post-Classical World, Cambridge, Cambridge University Press, 2014, p. 202-216. 
ANDO Clifford, « Was Rome a Polis », Classical Antiquity, 18, 1999, p. 5-34.

ATHANASSIADI Polymnia, « Antiquité tardive : construction et déconstruction d'un modèle historiographique », Antiquité tardive, 14, 2006, p. 311-324.

BÖRM Henning, « Hydatius von Aquae Flaviae und dir Einheit des Römischen Reiches im 5. Jahrhundert ", dans B. Bleckmann \& T. Stickler (éd.), Griechische Profanhistoriker des fünften nachchristlichen Jahrhunderts, Stuttgart, Franz Steiner, 2014, p. 195-214.

BÖRM Henning \& LURAGHI Nino (éd.), The Polis in the Hellenistic World, Stuttgart, Franz Steiner, 2018.

BURGESS Richard W. \& KULIKOWSKI Michael, Mosaics of Time. The Latin Chronicle Traditions from the First Century BC to the Sixth Century AD, vol. I : A Historical Introduction to the Chronicle Genre from the Origins to the High Middle Ages, Turnhout, Brepols, 2013.

DIAMOND Jared, Effondrement. Comment les sociétés décident de leur disparition ou de leur survie, Paris, Gallimard, 2006 [2005] (pagination de l'édition de poche Folio, 2009).

HANSEN Mogens, The Return of the Polis: The Use and Meanings of the Word Polis in Archaic and Classical Sources (Papers from the Copenhagen Polis Centre, vol. 8), Stuttgart, Franz Steiner, 2007.

INGLEBERT Hervé, Le Monde, L'Histoire. Essai sur les histoires universelles, Paris, PUF, 2014.

IOGNAT-PRAT Dominique, La Maison Dieu. Une histoire monumentale de l'Église au Moyen Âge, v. 800v. 1200, Paris, Le Seuil, 2006.

KULIKOWSKI Michael, «The Late Roman City in Spain », dans J.-U. Krause \& C. Witschel (éd.), Die Stadt in der Spätantike - Niedergang oder Wandel?, Stuttgart, Franz Steiner, 2006, p. 129-149.

PAWLAK Marcin, « Hydace et le désordre de son temps », Electum, 13, 2007, p. 29-37.

RAPP Claudia \& DRAKE Harold A. (éd.), The City in the Classical and Post-Classical World, Cambridge, Cambridge University Press, 2014.

RAPP Claudia \& DRAKE Harold A., «Polis-Imperium-Oikoumene : A World Reconfigured », dans C. Rapp \& H. A. Drake (éd.), The City in the Classical and Post-Classical World, Cambridge, Cambridge University Press, 2014, p. 1-13.

RATTI Stéphane (éd.), Une Antiquité tardive noire ou heureuse? (Actes du colloque international de Besançon, 12-13 novembre 2014), Besançon, Presses universitaires de Franche-Comté, 2015.

TRANOY Alain, La Galice romaine. Recherches sur le nord-ouest de la péninsule Ibérique dans l'Antiquité, Talence, Université Bordeaux 3, Centre Pierre Paris, diff. De Boccard, 1981.

\section{NOTES}

1. Résumé du rapport des Romains à l'espace : voir Albu (2014, 203-206).

2. Depuis l'édition française avec commentaire d'Alain Tranoy aux Sources chrétiennes en 1974, les travaux en français consacrés à Hydace ont été assez rares, alors que des avancées significatives sont à noter, en particulier du côté des historiographies anglo-saxonne et allemande, avec notamment Burgess (1993) et Scötter \& Scardino (2019), mais aussi des études plus spécifiques comme Börm (2014), ou Pawlak (2007). Un bon indicateur du dynamisme actuel des études hydaciennes pourrait être la comparaison des fiches Wikipedia se rapportant à Hydace : les fiches « latines » (français-italien-espagnol) sont très en retrait en termes de taille et de bibliographie par rapport aux fiches allemande et anglaise, elles-mêmes indépendantes l'une de l'autre (février 2020). 
3. Praefatio, 1 : Ydatius prouinciae Galleciae, natus in Lemica ciuitate.

4. Résumé des positions dans Athanassiadi (2006), voir aussi Ratti (2015). Jared Diamond (2006, 16) définit ainsi l'effondrement civilisationnel comme « une réduction drastique de la population humaine et/ou de la complexité politique/économique/sociale, sur une zone étendue et une durée importante ".

5. La Chronique exprime avec de multiples expressions cette idée de vivre des temps terribles et traumatisants (cf. Praefatio, 5-6), voir Pawlak (2007). Néanmoins, là où nous voyons aujourd'hui les signes de l'« effondrement » de la civilisation romaine, il semble qu'Hydace identifiait plutôt l'annonce d'un châtiment divin, voire celle de la fin des temps: c'est ce qui ressort de la référence implicite aux fléaux d'Ézéchiel 14:21 au $\S 40=$ M48. Cette dimension religieuse des événements politiques apparaît aussi avec la mention de prodiges effrayants et de mauvais augure : c'est tout particulièrement le cas pour 469 , année à laquelle s'interrompt sa chronique (\$ 246-247 = M252-253).

6. L'édition de référence a longtemps été celle de Mommsen dans les MGH, mais c'est désormais celle de Burgess (1993) qui fait autorité. L'édition française Tranoy (1974) quant à elle a souffert d'un excès de corrections : voir Burgess (1993, 27-31). Une toute nouvelle édition allemande vient aussi de paraître : Scötter \& Scardino (2019). C'est l'édition de Burgess que nous suivrons donc ici pour les références, avec une numérotation des chapitres différente de celle de Mommsen. Toutefois, comme la numérotation de Mommsen est encore couramment utilisée, j'indique aussi cette dernière quand c'est nécessaire : ainsi $\S 55=$ M67 signifie paragraphe 55 édition Burgess et 67 édition Mommsen.

7. Sur le genre de la chronique dans lequel se range l'œuvre d'Hydace, voir récemment Burgess \& Kulikowski (2013, 173-187) et Inglebert (2014, 409-418). Non uidi la thèse non publiée de Vivien Essoussi, "Hucusque Hieronymus": les continuateurs de la Chronique de saint Jérôme et la genèse de l'Occident post-romain au $v^{e}$ siècle. Étude historiographique, textes latins revus et traductions inédites, Paris 4, décembre 2011. Sur la question de savoir si Hydace a fait une chronique universelle ou pas, voir Scötter \& Scardino $(2019,18-22)$.

8. Praefatio, 1 : extremus plage; Praef., 6 : intra extremam universi orbis Gallaeciam. Alain Tranoy $(1981,453)$ parle en Galice d'une " civilisation "périphérique" ».

9. La question de la chronologie dans la Chronique et de ses incohérences est complexe, mais n'a pas vocation à être abordée ici.

10. La difficulté est en réalité masquée, car la simple transposition de ciuitas à " cité » semble intuitivement naturelle. Elle ressurgit en revanche quand on relève les autres équivalences données à " cité ». Il est ainsi frappant d'observer, dans le cas de la Chronique, la variété des choix de traduction de Richard Burgess pour rendre le mot urbs. En $\S 97=$ M106, il traduit par exemple eiusdem urbis par " of that same city "; en $§ 122=$ M130, in Asturicensi urbe Galleciae est traduit " in Asturica, a city of Gallaecia»: cela implique donc une équivalence ciuitas/urbs qui n'est qu'implicite. Pour un problème analogue, voir aussi la contribution dans ce dossier de Sabine Lefebvre consacrée à la traduction/interprétation d'oppidum chez César.

11. Sur polis grecque aux différentes époques, voir Hansen (2007) et Börm \& Luraghi (2018).

12. Cicéron, Les Lois, II, 5.

13. Ausone, Cortège des villes célèbres, v. 166 : haec patria est, patrias sed Roma superuenit omnes, «[Bordeaux] est ma patrie, mais Rome dépasse toutes les patries »; Rutilius Namatianus, Sur son retour, I, 1-34, 63-66. Sur ces questions de changement d'échelle, voir Rapp \& Drake (2014) qui intègrent en outre la dimension religieuse.

14. Voir les remarques de Rapp \& Drake $(2014,2)$ à propos de la traduction/interprétation du mot polis.

15. Ando (1999).

16. Rutilius Namatianus, Sur son retour, I, 19-34. Il y évoque les ciues des Gallica rura dont il se dit lui-même indigena. 
17. Un peu comme oppidum semble souvent désigner chez César une agglomération urbaine, mais prise uniquement dans sa dimension militaire et qu'on pourrait rendre par notre expression moderne "place militaire ", indépendamment de la présence de fortifications ou pas : voir les occurrences rassemblées dans ce même dossier par Sabine Lefebvre.

18. Rome : $355=\mathrm{M} 43 ; \S 36=\mathrm{M} 44 ; \S 120=\mathrm{M} 128 ; \S 130=\mathrm{M} 138 ; \S 155=\mathrm{M} 162 ; \S 211=\mathrm{M} 216$. Les autres occurrences désignent Constantinople $(\S 97=\mathrm{M} 106)$, Astorga $(\S 122=\mathrm{M} 130)$ et $\S 166=$ M173), Lugo ( $\$ 215=$ M219), Lerida ( $\$ 134=$ M142) ou l'ensemble des villes d'Hispanie ( $\$ 40=$ M48).

19. Praefatio, 1 : $2 ; \S 25=\mathrm{M} 31 ; \S 41=\mathrm{M} 49 ; \S 65=\mathrm{M} 73(2 \mathrm{x}) ; \S 79=\mathrm{M} 89 ; \S 142=\mathrm{M} 150(2 \mathrm{x})$; $\S 146=\mathrm{M} 154 ; \S 167=\mathrm{M} 174 ; \S 179=\mathrm{M} 186(2 \mathrm{x}) ; \S 181=\mathrm{M} 188 ; \S 201=\mathrm{M} 206 ; \S 210=\mathrm{M} 215 ; \S 237=$ M241; $\S 238=$ M244. On remarque aussi au passage que la seule mention de Constantinople la qualifie d'urbs.

20. $\S 179=$ M186 : residuis et uacuis ciuitatis domibus datis incendio, camporum loca uastantur.

21. Quant à savoir les raisons qui font qu'une ville comme Asturica est qualifiée à deux reprises par Hydace d'urbs mais jamais de ciuitas, elles restent un mystère.

22. Sur le maintien du rôle central de la ville en péninsule Ibérique à l'époque tardive et malgré l'effondrement romain, voir Kulikowski (2006).

23. Paulin de Pella, Poème d'action de grâces et prière, v. 25, 31, 37.

24. Iognat-Prat (2006).

25. Grégoire de Tours, Histoire des Francs, III, 19.

\section{RÉSUMÉS}

La Chronique d'Hydace permet de dresser une carte de géographie humaine centrée sur un espace très périphérique de l'Empire romain $d u v^{e}$ siècle apr. J.-C. Malgré sa vocation universelle, on y constate que le monde s'y réduit pour l'essentiel à l'espace ibérique dans le cadre plus large d'une géographie de plus en plus chrétienne. Ciuitas et urbs semblent y être interchangeables, mais certains indices pourraient indiquer un rôle plus important du premier terme.

Hydatius' Chronicle allows us to set a human geography map focused on the periphery of 5th century Roman Empire. Despite the pretention to be universal, it shows a world shrunk to Iberian territories as parts of the greater Christian space. Ciuitas and urbs seem to be interchangeable, but some evidence indicate that the first word is more significant.

\section{INDEX}

Mots-clés : géographie humaine, cité, ville, christianisation

Keywords : human geography, city, town, Christianization 
AUTEUR

CHRISTIAN STEIN

Université Bourgogne Franche-Comté

christian.stein@u-bourgogne.fr 